[2] Micu MC, Fodor D. Musculoskeletal ultrasound guided interventional manoeuvres. Musculoskeletal Ultrasonography in Rheumatic Diseases. Ed. Springer 2015, ISBN 978-3-319-15723-8.

Disclosure of Interests: None declared

DOI: 10.1136/annrheumdis-2020-eular.1619

\section{AB0874 $\quad$ FUNCTIONALITY IN OSTEOARTHRITIC GAIT IS RELATED TO TREATMENT DECISION. A MULTIFACTORIAL ANALYSIS}

S. Tassani ${ }^{1}$, L. Tío ${ }^{2}$, F. Castro ${ }^{2,3}$, J. Monfort ${ }^{2,3}$, J. C. Monllau ${ }^{2,4}$, M. Á. GónzálezBallester $^{1,5}$, J. Noailly ${ }^{1}{ }^{1}$ Universitat Pompeu Fabra, BCN MedTech, DTIC, Barcelona, Spain; ${ }^{2}$ Municipal Institute of Medical Research, Barcelona, Spain; ${ }^{3}$ Hospital del Mar, Rheumatology Department, Barcelona, Spain; ${ }^{4}$ Hospital del Mar, Traumatology Department, Barcelona, Spain; ${ }^{5}$ Institució Catalana de Recerca i Estudis Avançats (ICREA), Barcelona, Spain

Background: Osteoarthritis $(\mathrm{OA})$ is a degenerative disease with complex underling mechanisms ${ }^{1-3}$. The interactions among several factors make the study of the disease very complex and often lead to different treatment, i.e. surgical or conservative, decisions for subjects clinically and radiographically similar. Recent explorations performed at the body level pointed out that macro-factors, like overweight or gait, can influence the development of the disease ${ }^{4}$. The number of related factors is high, and they are very likely to interact with each other. However, the literature lacks randomized and balanced studies to verify such effects of multiple factors ${ }^{5}$. Objectives: The aim of this work was to develop a multifactorial analysis to explore whether and how gait functionality and dynamics can be related to treatment decision. Methods: A multifactorial analysis of gait dynamics in OA subjects was developed. $81 \mathrm{OA}$ subjects, graded 2-3 in $\mathrm{KL}$, were selected based on 4 clinical factors: Gender (male - female), Age (60-67 - 68-75), BMI (25-29.9-30+) and Treatment (total knee replacement (TKR) - conservative treatment). Gait analysis was performed using 8 cameras BTS Smart-DX 700, 1.5 Mpixels $250 \mathrm{fps}$ and 2 force plates BTS P-6000 $500 \mathrm{~Hz}$ sampling (BTS S.p.A., Milan, Italy). Helen Hayes marker protocol with medial markers was used for the study. Each volunteer was asked to perform a minimum of 5 valid gait sequences. Functionality and dynamics parameters were measured.

Functionality: Velocity of gait and the time needed to perform a gait cycle were computed.

Dynamics: The reaction forces and torques at the ankles, knees and hips were computed through inverse dynamic analyses.

Analysis of variance was performed for the four factors described among the functionality and dynamics parameters.

Results: The multifactorial analysis showed that functionality values are more subjective to the studied factors than the dynamics ones.

Functionality seems to be directly related to the clinical treatment. Patients who selected TKR needed more time to make a step, spent more time in double stand position and walked slower $(p<0.002)$. Older subject also walked slower but this dependency seemed to variate with age (interaction between clinical treatment and age $-\mathrm{p}<0.02-$ Figure 1$)$.

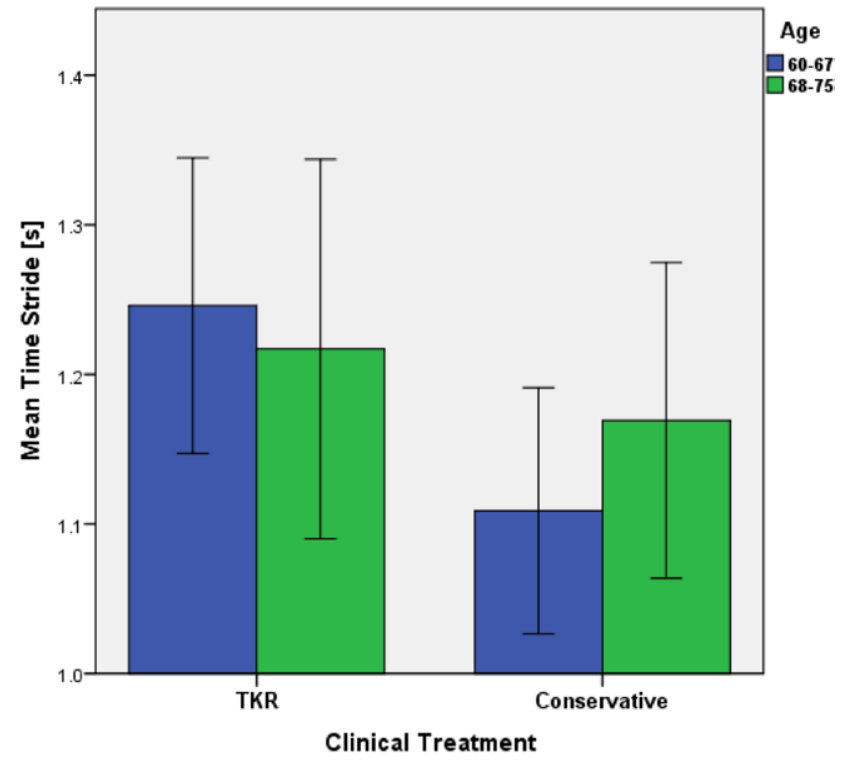

Error Bars: +/- 1 SD

Figure 1 Interaction between Clinical treatment and age.
Dynamics: Forces at the joints seemed to be affected by the gender and an interaction between age and $\mathrm{BMI}(\mathrm{p}<\mathrm{p}<0.005, \mathrm{p}<0.02)$ but not by the kind of therapy. Differently, torques were statistically related to the clinical treatment $(p<0.007)$. Age was also significant as was the interaction between age and BMI (both $\mathrm{p}<0.007$ )

Conclusion: Reduced functionality seems to be related to the selection of therapy. In contrast to current paradigm, forces at the joints may have no role in the definition of the best therapy for OA subjects. Subjects requiring TKR do not present higher loads at the joints. However, torques seems to be related to the therapy selected. Instead of forces, kinematics and posture assessments might support rational definitions of the therapy and future multifactorial analysis should take them into consideration.

References:

[1] Ding M. et al. J Bone Joint Surg Br. 2003;85(6):906-912.

[2] Kamibayashi L. et al. Calcif Tissue Int. 1995;57:69-73.

[3] Li B, Aspden RM. J Bone Miner Res. 1997;12(4):641-651.

[4] Berenbaum F et al. Curr Opin Rheumatol. 2013;25(1):114-118.

[5] Ioannidis JPA. Soc Sci Med. 2018;210(April):53-56.

Acknowledgments: MICINN Funds are acknowledged (HOLOA-DPI201680283-C2-1/2-R, RYC-2015-18888)

Disclosure of Interests: None declared

DOI: 10.1136/annrheumdis-2020-eular.1051

\section{$\mathrm{AB} 0875$ \\ HYBRID COOPERATIVE COMPLEXES OF SODIUM HYALURONATE + SODIUM CHONDROITIN NON- SULFATED (HA-SC) IN THE TREATMENT OF HIP OA: CLINICAL RESULTS}

R. Papalia ${ }^{1}$. ${ }^{1}$ Campus Bio-Medico University of Rome, Head of Department of Orthopaedics and Traumatology, Rome, Italy

Background: Hip Osteoarthritis (OA) is a widespread disease characterized by pain and functional impairment, which, particularly in the elderly, may compromise overall health and quality of life. In the last decades, Intra-articular (I.a.) injections of hyaluronic acid (HA) gained more space among the conservative treatment of $\mathrm{OA}$ because of their beneficial effects and positive outcomes without relevant complications. $(1,2)$. An innovative and patented formulation containing hybrid cooperative complexes of sodium hyaluronate $2.4 \%+$ sodium chondroitin non-sulfated $1.6 \%$ of biotechnological origin (HASC) has been recently developed for the I.a. treatment of hip OA and evaluated in a pilot study (3).

Objectives: 1) Primary aim: Evaluation of the safety of HA-SC in the treatment of symptomatic hip OA; 2) Secondary aim: Evaluation of the efficacy of HA-SC in terms of pain reduction (VAS) and function improvement (Lequesne) of the affected hip joint

Methods: This is a pilot, multicentric, open, prospective study. The following inclusion criteria were established: Patients (both genders) aged $\geq 40$ years suffering for primary hip OA confirmed by X-Ray; Grade I-II-III according to K\&L grading scale; Basal VAS pain at the target hip $>40 \mathrm{~mm}$; Failure of at least two lines of conservative treatments. All patients underwent a single I.a. hip injection of a $3 \mathrm{~mL}$ vial of HA-SC and followed-up for six months.

Results: 48 patients have been enrolled and completed the study. The treatment was generally well-tolerated, with only ten patients out of $48(20.8 \%)$ reporting local effects mainly consisting of injection site pain and arthralgia localized in the treated area. All these patients completed the study. The treatment with HA-SC was associated with a statistically significant decrease of VAS scale from a basal value of 67.5 (mean) to 22.8 (mean, $p<0.0001$ ) at the end of the observation period at six months, with a statistically significant decrease at seven days of follow-up evaluation $(29.3$, mean, $p<0.0001)$ The mean Lequesne's Index total score after the single injection of HA-SC decreased from a baseline value of 10.4 (mean) to 5.1 (mean, $\mathrm{p}<0.0001$ ) at six months. The decrease was marked and significant also at any of the other evaluated time point $(\mathrm{p}<0.0001)$.

Conclusion: A single I.a. injection of the innovative formulation containing hybrid cooperative complexes of sodium hyaluronate + sodium chondroitin non-sulfated (HA-SC) showed to be well tolerated and safe in the treatment of symptomatic hip OA. A rapid and significant decrease in hip pain (VAS) and Lequesne's Index was also observed starting immediately after the I.a. injection and lasting unti the end of the follow-up period. However, conservative treatment of hip OA is stil challenging. This new formulation could represent a promising, long-lasting, and effective I.a. treatment.

References:

[1] Papalia R. et al. J. Biol. Regul. Homeost. Agents 2017; 31 (4 Suppl. 2): 103-109.

[2] Abate M. et al. Int. J. Immunopathol.Pharmacol. 2017; 30 (1): 89-93.

[3] IBSA Data on file. 